\title{
Noncovalent Molecular Electronics
}

\author{
G. Gryn'ova and C. Corminboeuf*() \\ Institute of Chemical Sciences and Engineering, École polytechnique fédérale de Lausanne, CH-1015 Lausanne, Switzerland
}

\section{Supporting Information}

ABSTRACT: Molecular electronics covers several distinctly different conducting architectures, including organic semiconductors and single-molecule junctions. The noncovalent interactions, abundant in the former, are also often found in the latter, i.e., the dimer junctions. In the present work, we draw the parallel between the two types of noncovalent molecular electronics for a range of $\pi$-conjugated heteroaromatic molecules. In silico modeling allows us to distill the factors that arise from the chemical nature of their building blocks and from their mutual arrangement. We find that the same compounds are consistently the worst and the best performers in the two types of electronic assemblies, emphasizing the universal imprint of the underlying chemistry of the molecular cores on their diverse charge transport characteristics. The interplay between molecular and intermolecular factors creates a spectrum of noncovalent conductive architectures, which can be manipulated using the design strategies based upon the established relationships between chemistry and transport.

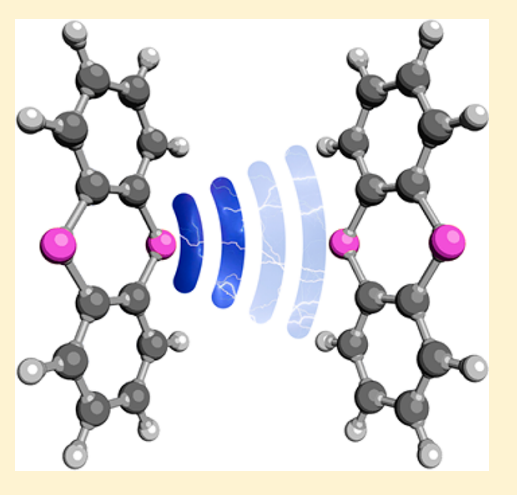

M olecular electronics (ME) is a broad and arguably blurry concept. There are in fact two principally different types-and communities-in ME: the sensu stricto unimolecular, or single-molecule, electronics and the sensu lato moleculebased electronics. Single-molecule junctions (SMJs) are a typical example of the former, while the latter refers to conducting crystals and polymers. ${ }^{1}$ Traditionally, a SMJ is thought of as an isolated molecule bridging the nanogap between two conducting (often metallic) electrodes, with the conductance of such an assembly being primarily attributed to the strength of the molecule-electrode coupling, the level alignment between the molecular orbitals and the electrodes' Fermi level(s), the presence of external electric fields and other environmental effects, etc. ${ }^{2}$ Recently, noncovalent interactions have started to attract growing attention as yet another factor influencing the SMJ transport properties. Some of the pioneering studies involved the $\pi$-stacked moieties incorporated into conducting wires ( 1 in Scheme 1) and attributed the observed high conductance of such junctions to an effective through-space $\pi, \pi$-coupling. ${ }^{3}$ In paracyclophane-based junctions (such as 2), conductance has been shown to drop exponentially with the number of $\pi$-stacked rings and to depend on how strained the cyclophane system is (type of linker connecting the rings) and on its symmetry (meta- vs para- connection). ${ }^{4}$ A similar trend in conductance decay with the number of rings was observed in the stacks of $\pi$-conjugated cores, locked within the self-assembled cages. ${ }^{5}$ Moreover, according to computational models, asymmetrically doping cyclophanes and nanographenes leads to SMJs with diodelike behavior (3 in Scheme 1). ${ }^{6}$ Beyond cyclophanes, the contribution of through-space transport has been demonstrated in conformationally flexible systems. Computational results by Ratner and et al. suggest that a 10 -fold increase in conductance can be expected for the $\pi$-stacked conformation of $\mathbf{4}$ formed upon elongation as compared to non- $\pi$-stacked conformers that exist at shorter electrode separations. ${ }^{7}$ In system $5, \pi$-stacking in a folded conformation compensates for the partially disrupted $\pi$-conjugation and larger highest occupied molecular orbital-lowest unoccupied molecular orbital (HOMOLUMO) gap leading to a conductance similar to that of an extended fully conjugated conformer. ${ }^{8}$ These studies illustrate the occurrence of multichannel conductance, i.e., when, in addition to conventional through-bond transport, systems with intramolecular $\pi$-coupling also offer the through-space transport mechanism.

An even more intriguing scenario is the transport in the absence of continuous through-bond conductance channel(s). Calame and et al. elegantly demonstrated the existence of such a current in mechanically controllable break junctions of oligo(p-phenyleneethynylene) (OPE) wires: molecules carrying only one thiol linker and thus capable of attachment to only one of the two gold electrodes still afford conductive nanobridges by means of dimer formation. ${ }^{9}$ Nichols and et al. further demonstrated that monomer and dimer junctions lead to distinguishable, characteristic peaks in conductance histograms. ${ }^{10}$ Such noncovalent junctions are attractive targets because of their potential for molecular sensing (via guest complex formation) ${ }^{11}$ and suppressed thermal conductance (by $95 \%$ compared to covalent junctions). ${ }^{12}$ However, dimer junctions are by no means exotic and do not exceptionally require monolinked molecules to form (Scheme 2). Most molecules in SMJ studies generally include planar $\pi$-conjugated regions prone to non-negligible intermolecular noncovalent interactions. $^{13,14}$ Therefore, dimeric bridges are in fact

Received: March 30, 2018

Accepted: April 17, 2018

Published: April 17, 2018 
Scheme 1. Literature Examples of SMJs with Intramolecular Noncovalent Interactions ${ }^{a}$

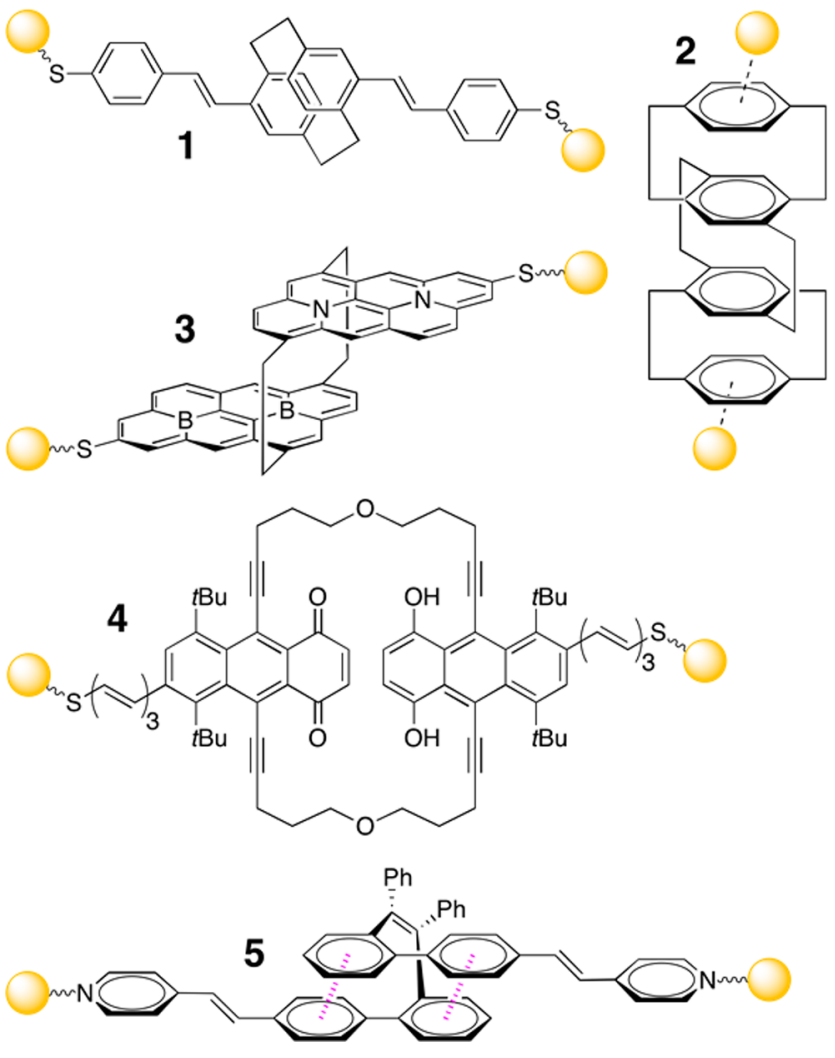

${ }^{a}$ Yellow spheres schematically represent the electrodes.

Scheme 2. Molecular Junction Scenarios: (A) Monomer Junction with Dilinked Core, (B) Dimer Junction with Dilinked Cores, and (C) Dimer Junction with Monolinked Cores

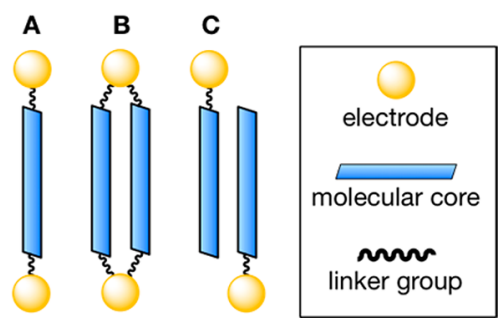

ubiquitous and can be observed in both simulations and experiments on many formally "single" molecule junctions. ${ }^{15-18}$

Transport through $\pi$-stacked systems has been rationalized using the concept of quantum interference that depends on the relative shifts between the $\pi$-stacked cores, their connectivity to the electrodes, and the substituent effects. ${ }^{19}$ Specifically, fully eclipsed dimers were found to be less conductive in the offresonant regime because of destructive quantum interferences that disappear once the cores are slightly shifted. More recently, Renaud et al. have shown that these periodic trends in $G / G_{0}$ versus lateral displacement between the cores in a dimer can be traced back to oscillations in the electronic coupling, $t$, between the frontier molecular orbitals (FMOs) of the cores. ${ }^{20}$ Periodicity of the $G / G_{0}$ behavior is thus dictated by the structure of the molecular cores and the phases of their highest occupied and/or lowest unoccupied molecular orbitals. Importantly, this trend mirrors the mobility in organic semiconductor crystals, in which the electronic coupling behavior is connected to the energetic characteristics of the noncovalent interactions between the neighbor cores. ${ }^{21}$

In the present work, we explore the similarities and differences in the properties of dimers of $\pi$-conjugated (hetero)aromatic cores between the two types of molecular electronics. Given the aforementioned connection of various features in both the molecular junctions and organic semiconductors to the chemical nature of the cores and the electronic couplings between them, we pose and address the following question: Would the molecular core, predicted to form a semiconductor with the highest charge mobility, also give the most conductive dimer junction?

We chose nine $\pi$-conjugated cores that are the common motifs in organic electronics (Scheme 3). ${ }^{22}$ Geometries of these

Scheme 3. Investigated Molecular Cores and Their Colors and Abbreviations Used Throughout This Letter ${ }^{a}$

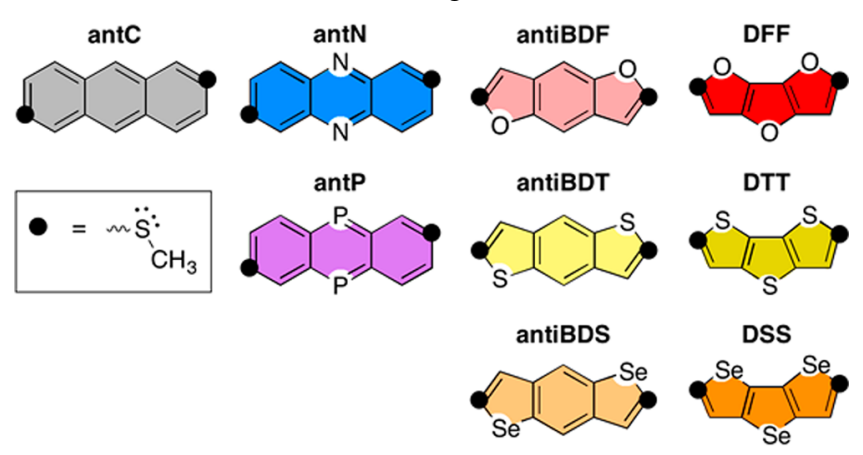

${ }^{a}$ See Figure $S 1$ in the Supporting Information for details. Black circles denote positions of the linker groups.

monomers were optimized at the PBE0/def2-SVP level. To form the monomer molecular junctions, the cores were flanked with the methylthio linkers, $-\mathrm{SCH}_{3}$, which are common functional groups in SMJs affording well-defined anchoring to the gold electrodes. ${ }^{23}$ Attachment positions in the cores (Scheme 3) were chosen to afford communication between the sites via the $\pi$-system and maintain the orbital symmetry of the cores (see Figure S1 in the Supporting Information). In the monomer junctions, each core was attached to the gold electrodes by its two linkers. Furthermore, for each core, two types of cofacial dimer junctions were considered: the so-called "constrained" and "optimized" dimers. The constrained dimer features a perfectly $\pi$-stacked (i.e., with zero longitudinal and transversal shifts, pitch, yaw, and roll angles) arrangement of two cores at a $3.75 \AA$ distance from each other (see Figure S2). In its junction, one core is connected covalently to one electrode and noncovalently to the other core, which in turn is connected via its linker to the opposite electrode. In the second, optimized, dimer type, the geometry of the dimer was relaxed between the two model $\mathrm{Au}_{8}$ anchors at the PBE0$\mathrm{D} 3 \mathrm{BJ} /$ def2-SVP level. In all cases, the nanogap was set to afford similar and optimal linker geometries. For both the monomers and the dimers, we have modeled their corresponding molecular junctions between the $\mathrm{Au}(111)$ electrodes. Transport properties of these junctions were evaluated using the nonequilibrium Green's functions formalism combined with the density functional theory (NEGF-DFT). ${ }^{24}$ Transmission peak alignments, computed at the PBE/DZP level of theory, are in a good agreement with the PBE0/def2-SVP orbital energy levels 
(Figure S4); importantly, only the qualitative trends are discussed in this work because most DFT methods struggle to accurately predict orbital energy levels. ${ }^{25}$ Molecular semiconductor charge mobility $(\mu)$ in the constrained and optimized dimers of the bare cores was evaluated using the Marcus-Hush theory ${ }^{26}$ based on the $\omega \mathrm{B} 97 \mathrm{X}-\mathrm{D} / \mathrm{maug}$-cc$\mathrm{pVTZ//PBE0/def2-SVP} \mathrm{reorganization} \mathrm{energy}(\lambda)$ of the monomer and the PBE0-dDsC ${ }^{27} / \mathrm{DZP}$ electronic coupling $(t)$ in the dimer. In all computations, appropriate corrections were introduced on heavy atoms to account for relativistic effects. A detailed description and discussion of these procedures is provided in the Supporting Information.

Selected (hetero)aromatic cores (Scheme 3) span three periods of the periodic table providing an insight into the role of atomic composition in the transport properties. All of them are fully conjugated, flat, and tricyclic and are therefore comparable to each other. For each core, two types of dimersconstrained in a perfect $\pi$-stack and relaxed-were considered. Their performance as conductive bridges in molecular junctions and as building blocks in bulk molecular semiconductors was evaluated with the ultimate aim of drawing parallels between these two types of molecular electronics.

Junction versus Semiconductor. Investigated junctions operate in the coherent off-resonant tunneling regime (see Figure S3); thus, their conductance $G / G_{0}$ exponentially decays with the nanogap length $L$, e.g., $G / G_{0} \sim \exp (-\beta L)$, where $\beta$ is the socalled decay constant. Lower decay constants correspond to higher conductance: they vary from $2.0 \AA^{-1}$ in vacuum to $0.7-$ $0.9 \AA^{-1}$ in alkanes and $0.05-0.2 \AA^{-1}$ in $\pi$-conjugated molecules. ${ }^{28}$ Because the junctions in our study have different nanogaps (see Table S1), we compare them using $\beta$ rather than the $G / G_{0}$ value at $E_{\mathrm{F}}$. For the organic semiconductor properties, constrained and optimized dimers are used as the simplest models, which are admittedly crude but insightful, ${ }^{29}$ and are compared in terms of their hole and electron mobility. The normalized trends in the computed transport properties in Figure 1 reveal several key features: (i) The constrained dimer junctions are rather similar to the monomer ones, but the optimized junctions deviate and strongly vary in a broader range of $\beta$. (ii) There is a noticeable agreement in the $\beta$ and $\mu$ trends. (iii) Strikingly, the same systems-antP and DFF-are consistently among the best and worst performers, respectively.

Molecular Factors. The similarity between the monomer and constrained dimer junctions suggests they are governed by molecular (rather than intermolecular, absent in the monomer junctions) factors. In a coherent off-resonant transport regime, the zero-bias conductance of a junction is largely determined by the tails of the nearby molecular orbital resonance peaks: closer peaks typically lead to larger tails around $E_{\mathrm{F}}$ and, ultimately, higher $G / G_{0}$ values. Indeed, we observe a robust correlation between the peak positions with respect to the Fermi level $\left(\Delta_{\text {peak }}\right)$ and transmission (i.e., the decay constants $\beta$ ) of the investigated junctions (Figure $2 \mathrm{~A}$ ). In organic semiconductors, reorganization energy is the key molecular factor defining their charge mobility: higher $\lambda$ results in lower mobility $\mu$ (Figure 2B).

Thus, there exist two molecular factors-energy of the molecular orbital resonance $\Delta_{\text {peak }}$ and reorganization energy $\lambda$-that play a definitive role in the charge transport of molecular junctions and molecular semiconductors, correspondingly. These two factors are not directly correlated with each other, but they are connected via the ionization potential (IP) and electron affinity (EA). Specifically, because trans-
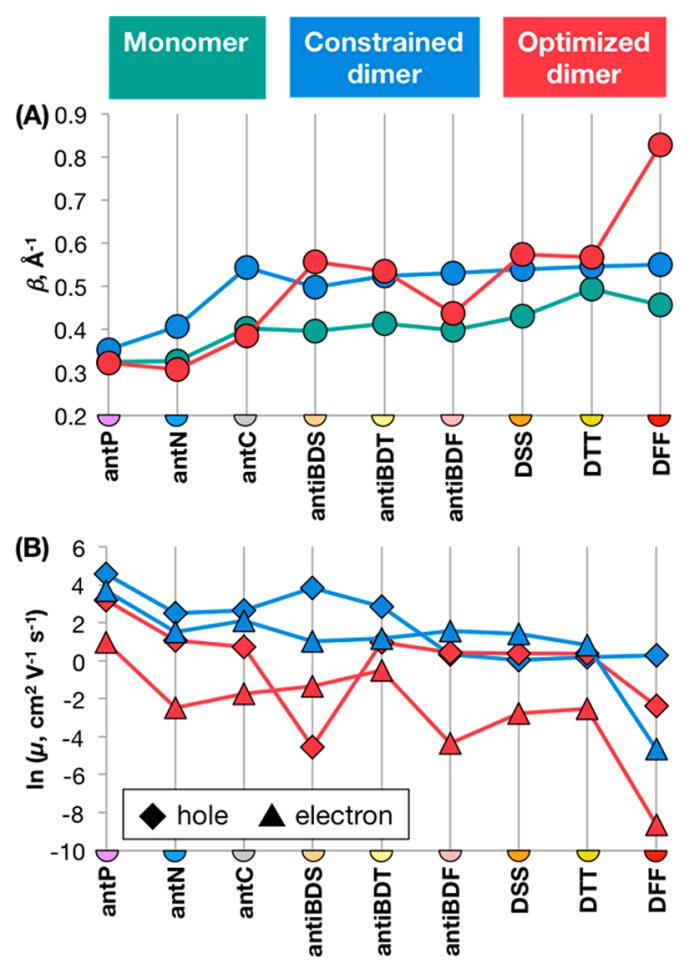

(C)

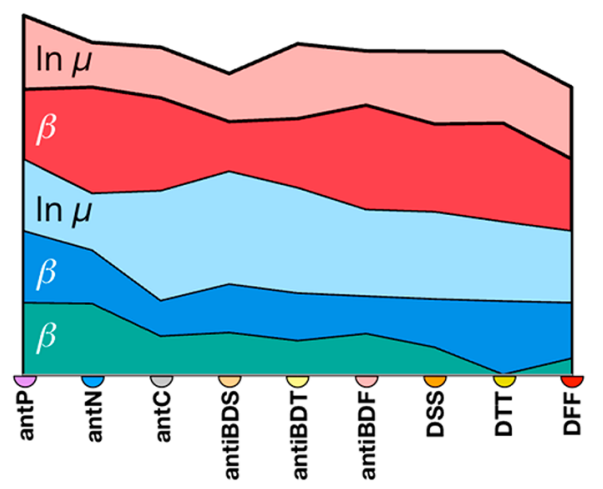

Figure 1. (A) Decay constants $\beta$ of the studied molecular junctions, computed from their NEGF-DFT conductance at the Fermi level. (B) Trends in computed electron and hole mobility $(\mu$, on a natural logarithm scale) of organic semiconductors, estimated using the constrained and optimized dimer models. (C) Relative trends in the normalized computed transport properties of molecular junctions and semiconductors. $\beta$ is normalized on a $0-1$ scale; maximum $\mu$, on $1-0$ scale; $y$-axis is qualitative only.

mission peaks correspond to the resonances of the conductive leads with the frontier molecular orbitals of the bridge, $\Delta_{\text {peak }}$ values are defined by the MO energy levels of the latter. ${ }^{30}$ In the spirit of Koopmans' (or Janak's) theorem, these HOMO and LUMO energy levels are reflected by the ionization potential and electron affinity, respectively. As a result, there is a strong correspondence between the vertical IP and position of a transmission peak, corresponding to a HOMO resonance, and vertical EA and $\Delta_{\text {peak LUMO }}$ (see Figure S5). Reorganization energy $\lambda$ is connected with IP and EA via electronegativity, i.e., Pearson's hardness equal to (IP - EA)/2 (see Figure S5). Thus, despite not being directly related, $\lambda$ and $\Delta_{\text {peak }}$ tend to be more favorable, thus leading to consistently better transport properties, for easily ionizable systems than for more electronically rigid ones. However, the correlations in Figure 2 are noticeably weaker in the optimized dimers, suggesting 


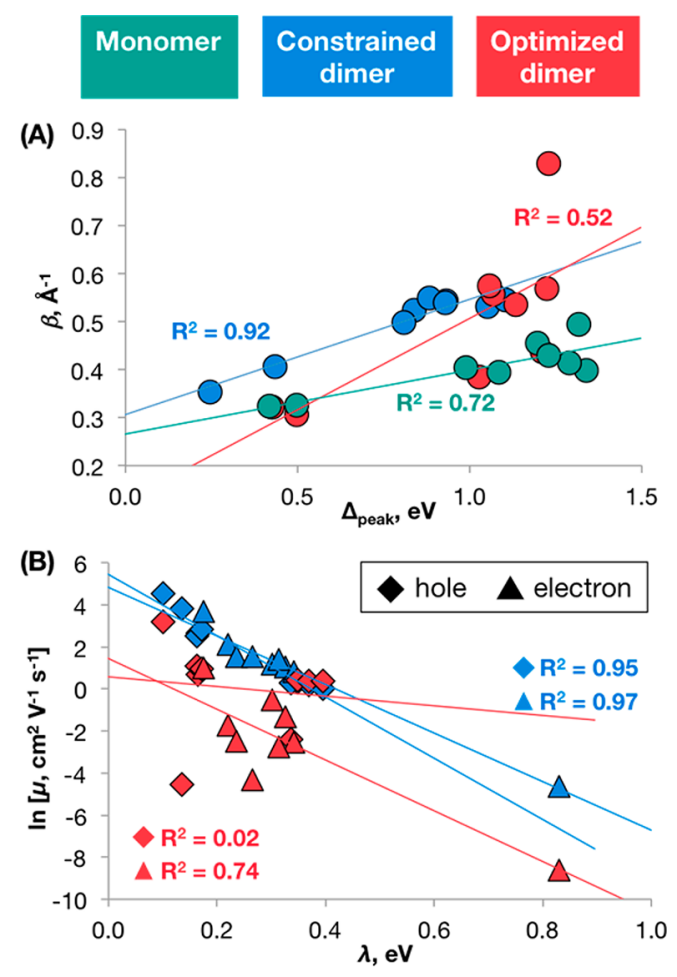

Figure 2. (A) Correlation between the decay constants $\beta$ and peak proximities $\Delta_{\text {peak }}$ (linear regression fits). (B) Correlation between the charge mobility $\mu$ and reorganization energy $\lambda$ (linear regression fits).

that additional influences on the charge transport arise in these systems.

Intermolecular Factors. Intermolecular orbital overlap is the major factor in the "communication" between the monomer cores within the dimer, impacting its charge-transporting properties. In the dimeric molecular junctions, the orbitals of the monomers split, leading to dual transmission peaks, ${ }^{31}$ with larger splitting $J$ originating from better intermolecular orbital overlap, i.e., higher electronic coupling (transfer integral) $t$ between the monomer cores (Figure 3A). We have already demonstrated that the peak proximity, $\Delta_{\text {peak }}$, has a determining effect on the transmission of the constrained dimers, in which the orbital overlap is maximized. Peak alignment is also critical in the optimized dimers of the anthracene-based cores. However, in the chalcogen-containing optimized dimers, $\Delta_{\text {peak }}$ is rather similar, and as a result, their transport is affected by the orbital energy level splitting; that is, within each family, dimers with higher $J$ have better conductivity (lower decay constant $\beta$, Figure $3 \mathrm{~B})$. In the context of organic semiconductors, higher electronic coupling $t$ leads to higher charge mobility (Figure 3C). Among the studied systems, dimers of antP possess some of the highest electronic couplings for both the hole and electron transfer, while DFF dimers have some of the lowest $t_{+}$ and $t_{-}$(Figure S6), and this is reflected in their charge mobility (Figure 1).

Therefore, the extent of intermolecular orbital overlap is the unifying factor influencing transmission in dimer junctions (via the orbital energy level splitting) and mobility in molecular semiconductors (via the electronic coupling). This feature is extremely sensitive to the shapes and symmetries of the monomers' FMOs and the mutual arrangement of the cores. $^{29,32}$ The latter is rather dissimilar between the constrained and optimized dimers, leading to the differences in their transport properties despite the identical molecular factors. The increasing role of the intermolecular factors in the charge transport of the optimized dimers is reflected by the somewhat stronger agreement in their $\beta$ and $\mu$ trends in Figure 1.

The Role of Chemistry. There exists a spectrum of relationships between the charge transport characteristics and the chemical features of the molecular building blocks (Figure 4 ). On one end of it are the perfectly $\pi$-stacked constrained dimers of different heteroaromatic cores, in which the electronic coupling is optimized to its maximum for a given interplanar separation. ${ }^{21}$ As a result, the transport efficiency of their electronic assemblies is less affected by this dimer property and is instead dominated by the more diverse parameters of the cores: reorganization energy and MO level

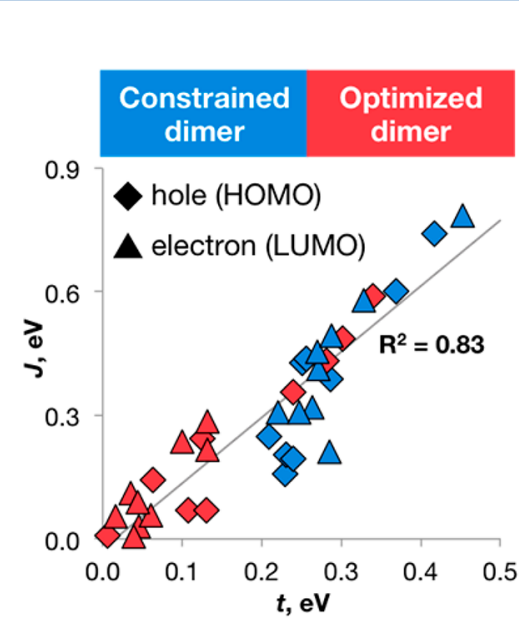

(A)

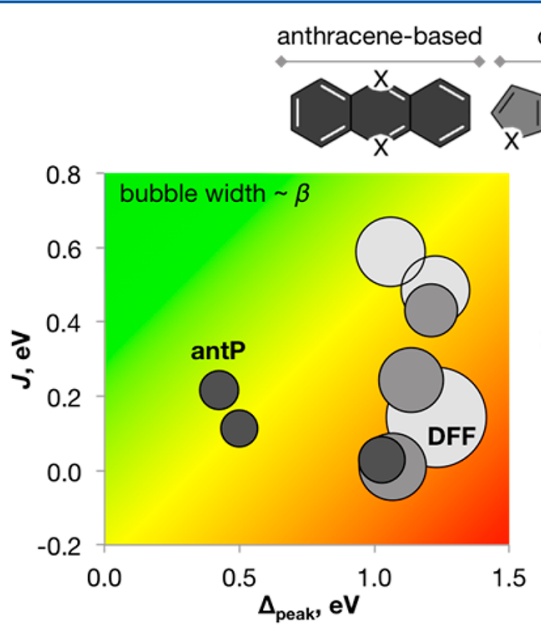

(B)
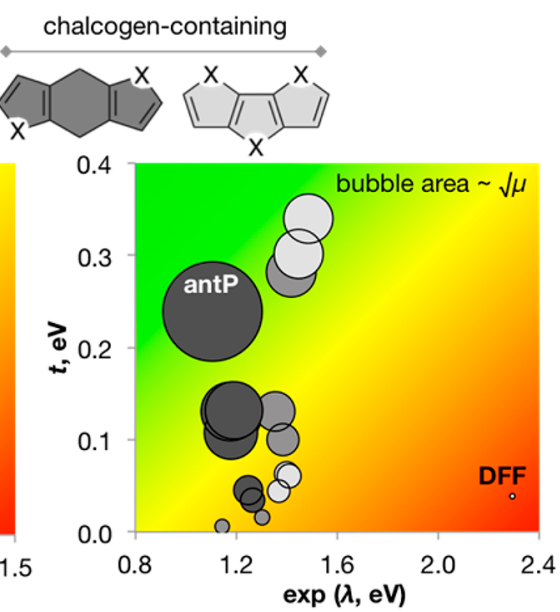

(C)

Figure 3. (A) Correlation between the computed electronic coupling $t$ and transmission peak splitting $J$. The straight line is the linear regression fit of all series combined. (B) Correlation between the computed characteristics of the optimized dimer junctions and their conductivity. (C) Correlation between the computed semiconductor characteristics of the optimized dimers and their charge mobility (hole and electron mobility combined). In panels B and C, the series are colored by the core family, and a red-to-green gradient qualitatively denotes improvement in the charge transport (lower $\beta$ and higher $\mu$ ). See Figure S7 in the Supporting Information for the constrained dimer plots. 


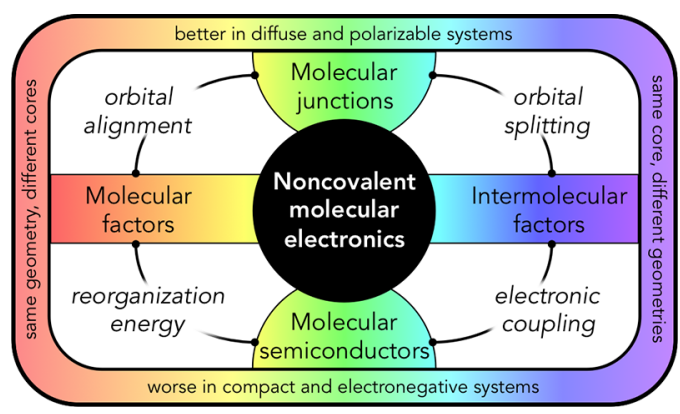

Figure 4. Spectrum of interplay between the molecular and intermolecular factors, determining the transport properties of various noncovalent molecular electronic architectures.

alignment with the $E_{\mathrm{F}}$. In the middle of the spectrum are the optimized dimers, whose electronic couplings are more spread out, leading to a broader range of charge transport characteristics and weaker correlations between $\beta$ and $\Delta_{\text {peak }}$ and between $\mu$ and $\lambda$ (Figure 2). At the opposite end of the spectrum are the geometrically different dimers of the same core, i.e., systems with identical $\lambda$ and $\Delta_{\text {peak }}$ but very different $t$ and $J$. In such junctions, conductance and mobility are uniquely defined by the latter, intermolecular parameters. ${ }^{20,29}$

The chemical nature of the cores, particularly their atomic composition, is a unifying factor across this spectrum: systems with diffuse and polarizable heteroatoms (e.g., phosphorus in phosphanthrene antP) consistently outperform systems with small, highly electronegative atoms (oxygen in difurofuran DFF). The former have low reorganization energies and lowlying unoccupied orbitals and also afford better intermolecular orbital overlap leading to higher electronic couplings. The latter, on the contrary, have wider HOMO-LUMO gap due to higher IP and/or lower EA and, being more compact, lead to weaker electronic couplings. Between these two extremes, a range of behavior exists because of an interplay of the aforementioned factors, e.g., low $\lambda$ and $\Delta_{\text {peak }}$ leading to efficient transport in antN dimers despite their average electronic couplings, or a high $\lambda_{+}$spoiling the performance of the DSS dimers notwithstanding the appreciable $t_{+}$. Established patterns can be employed to guide the molecular design of more efficient electronic assemblies by improving their molecular and/or intermolecular properties. As a proof-of-principle, we demonstrate this in Figure S8: introducing more phosphorus atoms to the anthracene skeleton reduces $\lambda$ and $-E A$ of the core and enhances the intermolecular orbital overlap in the dimer, thus improving its charge transport characteristics in the anthracene (zero P atoms) < phosphanthrene (two P atoms) < benzobisdiphosphinine (four $\mathrm{P}$ atoms) series.

Realistic Factors. In the present work, we focus on the role of the chemical cores in the properties of their single-molecule and molecular-based electronics. Even at this basic level, we by necessity made some simplifications in comparing the cores with and without the anchor groups, focusing on cofacial dimers and thus neglecting other disordermers. ${ }^{33}$ Beyond that, there are, of course, higher-order factors in terms of both modeling and experiment that can significantly impact the computed (and measured) performance characteristics. ${ }^{29,34}$ In the single-molecule junctions, these include dynamic effects, i.e., a spread of structures contributing to the measured current, ${ }^{35}$ coverage and solvent effects, the defects on the electrode surface and its polarization, etc. ${ }^{36}$ In organic crystals, the presence of thermal and energetic disorder and trap sites ${ }^{37}$ and specificity of molecular packing, among other factors, ${ }^{38}$ can play a crucial role in defining the charge carrier mobility. All these cumulatively add to the complexity of in silico and in situ studies on molecular electronics. At the core of it, however, remains the chemistry of the principal building blockmolecular structure-which we have shown to impact on the molecular junctions and crystalline semiconductors alike.

In conclusion, molecular electronics is an umbrella covering several distinctly different conductive architectures. In the present work, we have drawn a parallel between the transport in the nanogap between metallic electrodes and transport in the bulk of an organic semiconductor. By computing and comparing the properties of the model dimer molecular junctions and dimer units in molecular semiconductors, we have revealed that, within the model approximations, the same systems (phosphanthrene and difurofuran) are consistently among the best and the worst performers, with similar principles guiding their performance. On the spectrum of various molecular architectures, there is interplay between the intrinsic properties of molecular cores, e.g., the reorganization energies and ionization potentials or electron affinities, and the properties of the noncovalent assemblies, such as electronic coupling. Ultimately, the fundamental electronic structure features of the molecular cores leave the unifying footprint on the diverse charge transport properties.

\section{ASSOCIATED CONTENT}

\section{S Supporting Information}

The Supporting Information is available free of charge on the ACS Publications website at DOI: 10.1021/acs.jpclett.8b00980.

Detailed description of the computational procedures, additional figures and charts, full set of results, and geometries of all studied species (PDF)

\section{AUTHOR INFORMATION}

\section{Corresponding Author}

*E-mail: clemence.corminboeuf@epfl.ch.

ORCID $\odot$

C. Corminboeuf: 0000-0001-7993-2879

Notes

The authors declare no competing financial interest.

\section{ACKNOWLEDGMENTS}

This work has received support from EPFL and funding from the European Union's Horizon 2020 research and innovation programme under the Marie Skłodowska-Curie Grant Agreement No. 701885 "Single Molecule Junctions with NonConventional Architectures, Crafted in silico" and from the European Research Council (ERC Grant 306528 COMPOREL "Large-Scale Computational Screening and Design of Highlyordered pi-conjugated Molecular Precursors to Organic Electronic").

\section{REFERENCES}

(1) Metzger, R. M. Unimolecular Electronics. J. Mater. Chem. 2008, 18 (37), 4364-4396.

(2) Su, T. A.; Neupane, M.; Steigerwald, M. L.; Venkataraman, L.; Nuckolls, C. Chemical Principles of Single-Molecule Electronics. Nature Rev. Mater. 2016, 1, 16002.

(3) Seferos, D. S.; Trammell, S. A.; Bazan, G. C.; Kushmerick, J. G. Probing $\pi$-Coupling in molecular junctions. Proc. Natl. Acad. Sci. U. S. A. 2005, 102 (25), 8821-8825. 
(4) (a) Schneebeli, S. T.; Kamenetska, M.; Cheng, Z.; Skouta, R.; Friesner, R. A.; Venkataraman, L.; Breslow, R. Single-Molecule Conductance through Multiple $\pi$ - $\pi$-Stacked Benzene Rings Determined with Direct Electrode-to-Benzene Ring Connections. J. Am. Chem. Soc. 2011, 133 (7), 2136-2139. (b) Batra, A.; Kladnik, G.; Vázquez, H.; Meisner, J. S.; Floreano, L.; Nuckolls, C.; Cvetko, D.; Morgante, A.; Venkataraman, L. Quantifying Through-Space Charge Transfer Dynamics in $\pi$-Coupled Molecular Systems. Nat. Commun. 2012, 3, 1086. (c) Li, X.; Staykov, A.; Yoshizawa, K. Orbital Views on Electron-Transport Properties of Cyclophanes: Insight into Intermolecular Transport. Bull. Chem. Soc. Jpn. 2012, 85 (2), 181-188. (d) Bai, M.; Liang, J.; Xie, L.; Sanvito, S.; Mao, B.; Hou, S. Efficient Conducting Channels Formed by the $\pi-\pi$ Stacking in Single [2,2]Paracyclophane Molecules. J. Chem. Phys. 2012, 136 (10), 104701.

(5) Kiguchi, M.; Takahashi, T.; Takahashi, Y.; Yamauchi, Y.; Murase, T.; Fujita, M.; Tada, T.; Watanabe, S. Electron Transport through Single Molecules Comprising Aromatic Stacks Enclosed in SelfAssembled Cages. Angew. Chem., Int. Ed. 2011, 50 (25), 5708-5711.

(6) Staykov, A.; Li, X.; Tsuji, Y.; Yoshizawa, K. Current Rectification in Nitrogen- and Boron-Doped Nanographenes and Cyclophanes. J. Phys. Chem. C 2012, 116 (34), 18451-18459.

(7) Franco, I.; Solomon, G. C.; Schatz, G. C.; Ratner, M. A. Tunneling Currents That Increase with Molecular Elongation. J. Am. Chem. Soc. 2011, 133 (39), 15714-15720.

(8) Chen, L.; Wang, Y.-H.; He, B.; Nie, H.; Hu, R.; Huang, F.; Qin, A.; Zhou, X.-S.; Zhao, Z.; Tang, B. Z. Multichannel Conductance of Folded Single-Molecule Wires Aided by Through-Space Conjugation. Angew. Chem., Int. Ed. 2015, 54 (14), 4231-4235.

(9) Wu, S.; González, M. T.; Huber, R.; Grunder, S.; Mayor, M.; Schönenberger, C.; Calame, M. Molecular Junctions Based on Aromatic Coupling. Nat. Nanotechnol. 2008, 3, 569-574.

(10) Martin, S.; Grace, I.; Bryce, M. R.; Wang, C.; Jitchati, R.; Batsanov, A. S.; Higgins, S. J.; Lambert, C. J.; Nichols, R. J. Identifying Diversity in Nanoscale Electrical Break Junctions. J. Am. Chem. Soc. 2010, 132 (26), 9157-9164.

(11) Das, B.; Abe, S. Designing Cyclophane-Based Molecular Wire Sensors. J. Phys. Chem. B 2006, 110 (47), 23806-23811.

(12) Li, Q.; Strange, M.; Duchemin, I.; Donadio, D.; Solomon, G. C. A Strategy to Suppress Phonon Transport in Molecular Junctions Using $\pi$-Stacked Systems. J. Phys. Chem. C 2017, 121 (13), 71757182 .

(13) Wheeler, S. E.; Bloom, J. W. G. Toward a More Complete Understanding of Noncovalent Interactions Involving Aromatic Rings. J. Phys. Chem. A 2014, 118 (32), 6133-6147.

(14) Sutton, C.; Risko, C.; Brédas, J.-L. Noncovalent Intermolecular Interactions in Organic Electronic Materials: Implications for the Molecular Packing vs Electronic Properties of Acenes. Chem. Mater. 2016, 28 (1), 3-16.

(15) Ghane, T.; Nozaki, D.; Dianat, A.; Vladyka, A.; Gutierrez, R.; Chinta, J. P.; Yitzchaik, S.; Calame, M.; Cuniberti, G. Interplay between Mechanical and Electronic Degrees of Freedom in $\pi$-Stacked Molecular Junctions: From Single Molecules to Mesoscopic Nanoparticle Networks. J. Phys. Chem. C 2015, 119 (11), 6344-6355.

(16) Yoshida, R.; Pobelov, I. V.; Manrique, D. Z.; Pope, T.; Mészáros, G.; Gulcur, M.; Bryce, M. R.; Lambert, C. J.; Wandlowski, T. Correlation of Breaking Forces, Conductances and Geometries of Molecular Junctions. Sci. Rep. 2015, 5, 9002.

(17) Lemmer, M.; Inkpen, M. S.; Kornysheva, K.; Long, N. J.; Albrecht, T. Unsupervised Vector-Based Classification of SingleMolecule Charge Transport Data. Nat. Commun. 2016, 7, 12922.

(18) Magyarkuti, A.; Adak, O.; Halbritter, A.; Venkataraman, L. Electronic and Mechanical Characteristics of Stacked Dimer Molecular Junctions. Nanoscale 2018, 10 (7), 3362-3368.

(19) (a) Solomon, G. C.; Herrmann, C.; Vura-Weis, J.; Wasielewski, M. R.; Ratner, M. A. The Chameleonic Nature of Electron Transport through $\pi$-Stacked Systems. J. Am. Chem. Soc. 2010, 132 (23), 78877889. (b) Solomon, G. C.; Vura-Weis, J.; Herrmann, C.; Wasielewski, M. R.; Ratner, M. A. Understanding Coherent Transport through $\pi$ -
Stacked Systems upon Spatial Dislocation. J. Phys. Chem. B 2010, 114 (45), 14735-14744. (c) Li, Q.; Solomon, G. C. Exploring Coherent Transport through $\pi$-Stacked Systems for Molecular Electronic Devices. Faraday Discuss. 2014, 174, 21-35.

(20) Frisenda, R.; Janssen, V. A. E. C.; Grozema, F. C.; van der Zant, H. S. J.; Renaud, N. Mechanically Controlled Quantum Interference in Individual $\pi$-Stacked Dimers. Nat. Chem. 2016, 8, 1099-1104.

(21) Gryn'ova, G.; Corminboeuf, C. Implications of Charge Penetration for Heteroatom-Containing Organic Semiconductors. J. Phys. Chem. Lett. 2016, 7 (24), 5198-5204.

(22) (a) Anthony, J. E. The Larger Acenes: Versatile Organic Semiconductors. Angew. Chem., Int. Ed. 2008, 47, 452-483. (b) Takimiya, K.; Nakano, M.; Sugino, H.; Osaka, I. Design and Elaboration of Organic Molecules for High Field-Effect-Mobility Semiconductors. Synth. Met. 2016, 217, 68-78. (c) Dong, H.; Fu, X.; Liu, J.; Wang, Z.; Hu, W. 25th Anniversary Article: Key Points for High-Mobility Organic Field-Effect Transistors. Adv. Mater. 2013, 25 , 6158-6183. (d) Baumgartner, T. Insights on the Design and ElectronAcceptor Properties of Conjugated Organophosphorus Materials. Acc. Chem. Res. 2014, 47, 1613-1622.

(23) Leary, E.; La Rosa, A.; González, M. R.; Rubio-Bollinger, G.; Agrait, M.; Martin, N. Incorporating Single Molecules into Electrical Circuits. The Role of the Chemical Anchoring Group. Chem. Soc. Rev. 2015, 44, 920-942.

(24) (a) Maassen, J.; Harb, M.; Michaud-Rioux, V.; Zhu, Y.; Guo, H. Quantum Transport Modeling From First Principles. Proc. IEEE 2013, 101 (2), 518-530. (b) Quek, S. Y.; Khoo, K. H. Predictive DFT-Based Approaches to Charge and Spin Transport in Single-Molecule Junctions and Two-Dimensional Materials: Successes and Challenges. Acc. Chem. Res. 2014, 47 (11), 3250-3257. (c) Seldenthuis, J. S. Electrical and Mechanical Effects in Single-Molecule Junctions. Ph.D. Thesis, TU Delft, 2011. (d) Verzijl, C. J. O.; Thijssen, J. M. DFTBased Molecular Transport Implementation in ADF/BAND. J. Phys. Chem. C 2012, 116 (446), 24393-24412.

(25) (a) Zhang, G.; Musgrave, C. B. Comparison of DFT Methods for Molecular Orbital Eigenvalue Calculations. J. Phys. Chem. A 2007, 111 (8), 1554-1561. (b) Brédas, J.-L. Mind the gap! Mater. Horiz. 2014, 1 (1), 17-19.

(26) (a) Marcus, R. A. Electron Transfer Reactions In Chemistry. Theory And Experiment. Rev. Mod. Phys. 1993, 65 (3), 599-610. (b) Barbara, P. F.; Meyer, T. J.; Ratner, M. A. Contemporary Issues in Electron Transfer Research. J. Phys. Chem. 1996, 100 (31), 1314813168.

(27) Steinmann, S. N.; Corminboeuf, C. Comprehensive Benchmarking of a Density-Dependent Dispersion Correction. J. Chem. Theory Comput. 2011, 7 (11), 3567-3577.

(28) Khoo, K. H.; Chen, Y.; Li, C.; Quek, S. Y. Length Dependence of Electron Transport Through Molecular Wires - a First Principles Perspective. Phys. Chem. Chem. Phys. 2015, 17 (1), 77-96.

(29) Gryn'ova, G.; Nicolaï, A.; Prlj, A.; Ollitrault, P.; Andrienko, D.; Corminboeuf, C. Charge Transport in Highly Ordered Organic Nanofibrils: Lessons from Modelling. J. Mater. Chem. C 2017, 5, 350361.

(30) (a) Tsuji, Y.; Hoffmann, R. Frontier Orbital Control of Molecular Conductance and its Switching. Angew. Chem., Int. Ed. 2014, 53 (16), 4093-4097. (b) Quinn, J. R.; Foss, F. W., Jr.; Venkataraman, L.; Breslow, R. Oxidation Potentials Correlate with Conductivities of Aromatic Molecular Wires. J. Am. Chem. Soc. 2007, 129 (41), 1237612377.

(31) Geng, H.; Yin, S.; Chen, K.-Q.; Shuai, Z. Effects of Intermolecular Interaction and Molecule-Electrode Couplings on Molecular Electronic Conductance. J. Phys. Chem. B 2005, 109 (25), 12304-12308.

(32) Coropceanu; Cornil, J.; da Silva Filho, D. A.; Olivier, Y.; Silbey, R.; Brédas, J.-L. Charge Transport in Organic Semiconductors. Chem. Rev. 2007, 107 (4), 926-952.

(33) Thorley, K. J.; Risko, C. On the Impact of Isomer Structure and Packing Disorder in Thienoacene Organic Semiconductors. J. Mater. Chem. C 2016, 4 (18), 4040-4048. 
(34) Jin, C.; Thygesen, K. S. Dynamical Image-Charge Effect in Molecular Tunnel Junctions: Beyond Energy Level Alignment. Phys. Rev. B: Condens. Matter Mater. Phys. 2014, 89, 041102.

(35) Yoshida, S.; Taninaka, A.; Sugita, Y.; Katayama, T.; Takeuchi, O.; Shigekawa, H. Revealing the Conformational Dynamics in a SingleMolecule Junction by Site- and Angle-Resolved Dynamic Probe Method. ACS Nano 2016, 10 (12), 11211-11218.

(36) Xiang, D.; Wang, X.; Jia, X.; Lee, T.; Guo, X. Molecular-Scale Electronics: From Concept to Function. Chem. Rev. 2016, 116 (7), 4318-4440.

(37) Rühle, V.; Lukyanov, A.; May, F.; Schrader, M.; Vehoff, T.; Kirkpatrick, J.; Baumeier, B.; Andrienko, D. Microscopic Simulations of Charge Transport in Disordered Organic Semiconductors. J. Chem. Theory Comput. 2011, 7 (10), 3335-3345.

(38) Brédas, J. L.; Calbert, J. P.; da Silva Filho, D. A.; Cornil, J. Organic Semiconductors: A Theoretical Characterization of the Basic Parameters Governing Charge Transport. Proc. Natl. Acad. Sci. U. S. A. 2002, 99 (9), 5804-5809. 